\title{
Philippine Normal Reference Values for Cardiopulmonary Exercise Testing
}

\author{
Lenora Fernandez ${ }^{1}$, Camilo Roa $\mathbf{J r}^{1}$, Liza Llanes-Garcia ${ }^{2}$, Norman Maghuyop ${ }^{1}$ \\ ${ }^{1}$ Division of Pulmonary Medicine, University of the Philippines Philippine General Hospital, Manila City, Philippines \\ ${ }^{2}$ Section of Pulmonology, The Medical City, Pasig City, Philippines
}

Email address:

lcfernandez3@up.edu.ph (L. Fernandez)

\section{To cite this article:}

Lenora Fernandez, Camilo Roa Jr, Liza Llanes-Garcia, Norman Maghuyop. Philippine Normal Reference Values for Cardiopulmonary Exercise Testing. American Journal of Internal Medicine. Vol. 9, No. 2, 2021, pp. 70-75. doi: 10.11648/j.ajim.20210902.12

Received: February 16, 2021; Accepted: March 2, 2021; Published: March 9, 2021

\begin{abstract}
Performance on cardiopulmonary exercise test (CPET) is influenced by genetic, racial and environmental factors. This study aimed to establish the Maximum Working Capacity, Peak oxygen uptake $\left(\mathrm{VO}_{2}\right)$, Peak carbon dioxide output $\left(\mathrm{VCO}_{2}\right)$, Tidal Volume (TV) for normal Filipinos during exercise and derive prediction equation models for Maximum Working Capacity, Peak $\mathrm{VO}_{2}$, Peak $\mathrm{VCO}_{2}$ and maximum attained TV. The maximal cardiopulmonary responses were analyzed for one hundred eighteen healthy sedentary adult Filipino subjects who underwent CPET using a symptom limited incremental progressive cycle ergometer driven protocol. A Vista Mini-CPX Model 17670 was used. Models for predicting $\mathrm{VO}_{2} \mathrm{max}_{\mathrm{V}} \mathrm{VCO}_{2}$ peak, TV peak, and Work Max were derived with height, weight, age and sex being screened for significance as predictors. Linear and non-linear regression analyses were done. The maximum working capacity for males was $154.21 \pm 26.6$ watts and $93.02 \pm 15.57$ watts for females while the peak $\mathrm{VO}_{2}$ for males was $4.90 \pm 3.11$ and $4.56 \pm 2.41$ liters/minute for females. The predictive formulae derived from this study for maximum work capacity, peak $\mathrm{VO}_{2}, \mathrm{VCO}_{2}$ and tidal volume had acceptable correlation coefficients and performed as well as other published predictive equations based on Caucasian and Asian populations. Differences between races as to performance in exercise testing was confirmed in this study.
\end{abstract}

Keywords: Cardiopulmonary Exercise Test, Philippine Normal Reference Values

\section{Introduction}

Cardio-pulmonary exercise (CPX) is a recognized procedure in evaluating human exercise performance. In contrast to resting examinations of cardiovascular and pulmonary systems, CPX can better detect, quantify and differentiate cardiac and respiratory abnormalities even when resting examinations are normal [1]. In addition, it can provide the basis for evaluating subjective symptoms of dyspnea and fatigue [2]. It is being used to evaluate fitness, risk stratification, impairment and disability in the occupational setting. It forms the basis of activity or establishing guidelines for exercise prescriptions in rehabilitation programs [3].

A CPX system can also be used to monitor substrate metabolism for energy production and to define the response to therapy [3]. It is a valuable tool to study nutritional interventions, metabolic drug effects and other studies involving energy kinetics $[4,5]$. This set-up has been shown to be a useful aid for the understanding of cardiovascular, pulmonary and neuromuscular physiology by medical students [5].

To interpret each result, standard reference values taken from a normal population are used [3, 6, 7]. Human performance, however, is influenced by genetic, racial and environmental factors [2, 3]. Normal reference values derived from Caucasian populations are not deemed appropriate if CPX is done among non-Caucasian subjects $[3,6,7]$. It is important, therefore, to establish a local set of reference values for the Filipino population.

The risks to the patients of an exercise test are very small, provided that simple precautions are observed [8, 9]. The dangers of myocardial infarction or serious arrhythmia is estimated at about 1 in 10,000 tests [9, 10].

This study aimed to establish the normal cardio-pulmonary exercise values using an incremental progressive cycle ergometer driven protocol among healthy, sedentary adult Filipinos. The specific objectives were to establish Maximum 
Working Capacity, Peak oxygen intake $\left(\mathrm{VO}_{2}\right)$, Peak carbon dioxide output $\left(\mathrm{VCO}_{2}\right)$ and Tidal Volume (TV) for normal Filipinos during exercise. The median perceived rate of exertion using the Borg Scale was also determined. Prediction equation models for Maximum Working Capacity, Peak $\mathrm{VO}_{2}$, Peak $\mathrm{VCO}_{2}$ and maximum attained TV were then derived. The null hypothesis in this study was that prediction equation models for Maximum Working Capacity, Peak $\mathrm{VO}_{2}$, Peak $\mathrm{VCO}_{2}$ and maximum attained $\mathrm{TV}$ were similar to the prediction equation models for Caucasians.

\section{Methods}

\section{A. Selection \& Description of Participants}

This was a cross-sectional analytical study. Approval from the local ethics board was obtained. The target population of the study was obtained from the healthy adult population of Metro Manila and neighboring towns and cities. Subjects were recruited from hospital personnel and the general population in the neighboring cities and provinces by advertisement. The recruited subjects were stratified into 4 age groups (20-29, 30-39, 40-49, and 50-60) for both sexes. Informed consent was obtained from each subject before entry into the study. The subjects had to meet the following inclusion criteria: age between 20 and 60 years old with normal body mass index (BMI), physical examination findings, chest radiographic findings, 12-lead electrocardiogram, resting spirometry values, hemoglobin and hematocrit values, total smoking history of $<0.5$ pack years with no smoking for the past six months, and had signed the informed consent form. Subjects were excluded when they were considered competitive athletes based on history, pregnant, had history of chronic illness (obstructive lung disease, cardiovascular disease, musculoskeletal abnormalities, etc.), chest pains or electrocardiographic evidence of myocardial ischemia.

\section{B. Technical Information}

The following study equipment

The study equipment used were the Vista Mini-CPX Model 17670 with Turbo-Fit software, cycle Ergometer Lobe BV V2.57 Model, digital pulse oximeter, 12-lead ECG machine (ECG Cardioline Machine built-in to the CPX machine), cardiac monitor and defibrillator, aneroid sphygmomanometer, spirometer, oxygen, emergency drugs and resuscitation materials. These were provided by the Center for Enhancement of Human Performance Laboratory of the Department of Physiology, University of the Philippines College of Medicine, Pedro Gil Street, Manila.

Subjects who fulfilled the inclusion and exclusion criteria underwent spirometry just prior to the exercise test. A trained physical therapist determined FEV1, FVC, and FEV1/FVC, and bronchodilator response using Spirobank office spirometer and WinSpiro software version 2.36b (Medical International Research). The spirometry was done in the sitting position. All tests were done at the Pulmonary Laboratory of the Philippine General Hospital. Normal values for non Caucasians based on age, weight and height were derived using the formula by Knudson. Maximum voluntary ventilation (MVV) was computed based on FEV1. The spirometry results were interpreted by one of the authors. Subjects deemed to have normal spirometric results underwent the exercise test.

All exercise tests were performed at the Department of Physiology Center for Enhancement of Human Performance Laboratory. The subjects were instructed on suitable clothing and to come to the exercise laboratory at least 2 hours after their last meal. The Vista Mini-CPX Machine Model 17670 with metabolic monitor, computer, and Hans Rudolf valves and masks were used. This machine has a $\mathrm{VO}_{2}$ accuracy of $+/-3 \%$. The $\mathrm{O}_{2}$ and $\mathrm{CO}_{2}$ gas analyzers were calibrated prior to each use and every 3 months. Room temperature and humidity were adjusted at 23 to $28^{\circ} \mathrm{C}$ and 63 to $75 \%$, respectively. Tidal volume was reported at ambient temperature, and pressure, fully saturated with water (ATPS). The subjects were carefully instructed on how to perform the exercise and how it is terminated.

The supervising physician obtained the medical history and performed a complete physical examination on each subject. Anthropometric measurements (height in cms and weight in kgs) were made using standard techniques.

The electrocardiograph electrodes were correctly positioned and secured using self-adhesive, disposable, silver ECG electrodes. A sphygmomanometer cuff was placed on the upper arm, secured, and taped in place. The pulse oximeter was positioned to one of the subject's fingers.

The subject assumed a comfortable cycling position on the saddle. The knee was fully extended at the bottom of the pedal stroke and the handlebars were maintained at waist height. The metabolic machine facemask was positioned to create an airtight seal and so that the patient was leaning forward.

Following the Jones Protocol 5 for cycle ergometer, the patient was instructed to pedal at the initial load of 16 watts (100 kpm) [6]. At the end of each minute the power is increased by 16 watts (increments of $100 \mathrm{kpm}$ until patient stops because of symptoms, or because safety limits have been reached).

During the exercise testing, blood pressure was determined using a calibrated sphygmomanometer and was recorded during the last 15 seconds of each 3-minute intervals. Heart rate and cardiac rhythm were continuously monitored through a cardiac monitor and an ECG tracing was obtained at each workload. Oxygen saturation was continuously monitored by a pulse oximeter. A portable cardiac monitor and defibrillator was available throughout the entire duration of the test.

Expired air samples were collected through Hans-Rudolph valves to an automated gas analyzer for serial monitoring of respiratory rate (RR), tidal volume (TV), minute ventilation (VE), and $\mathrm{CO}_{2}$ production $\left(\mathrm{VCO}_{2}\right)$.

At the end of each minute, the subject was asked if he felt he can carry on and whether he had any significant symptoms. Quantitation of symptoms was done by the subject at the end of the peak exercise test using the Borg category scale.

The decision to stop an exercise test was dependent on an understanding of the patients' clinical situation. Criteria for discontinuation of the exercise at any stage were set. 2,3 
Maximal exercise was defined as fulfillment of at least one of following three criteria: 1) failure of $\mathrm{VO}_{2}$ to rise with increasing workload; 2) respiratory gas exchange ratio greater than $1.15 ; 3$ ) heart rate at least $85 \%$ of predicted maximal heart rate (HRmax) as determined by the following formula: HRmax $=220$ - age in years.

All parameters were also measured during the immediate recovery period. Additional measurements were recorded at 3 minutes, 5 \& 10 minutes after peak exercise while the patient remain seated on the cycle ergometer saddle.

The heart rate was determined from the ECG readings in the Cardioline Digital Electrocardiograph, and confirmed from the pulse oximeter. Respiratory variables (which include $\mathrm{VO}_{2}$, Maximal Power Output, $\mathrm{CO}_{2}$ output, Respiratory Rate, Respiratory Exchange Ratio, Tidal Volume) were measured and/or derived using the metabolic machine. The systolic and diastolic blood pressure were also measured using the auscultation technique at the last 15 seconds of each 3-minute interval.

\section{Statistics}

Models for predicting $\mathrm{VO}_{2} \max , \mathrm{VCO}_{2}$ peak, VT peak, and Work max were derived using the Statistical Package for the Social Sciences (SPSS). Automatic search procedure (stepwise) was used to derive the best model. Height, weight, age and sex were screened for significance as predictors of the said parameters. Preliminary investigation was done by analyzing scatter plots of the exercise parameters versus the candidate predictors. Linear and non-linear regression analyses were done. Dubin-Watson was used to check for auto-correlation. Multi linearity was tested using variance influence factor (VIF). The influences of outliers were tested using DFFITS. All derived models were tested for normality using the Kormoglov-Smirnoff test and were tested for equality of error variances using Leavene's test.

\section{Results}

One hundred forty nine volunteers were screened for this study. One patient had an abnormal chest x-ray. One patient was unable come to the scheduled day of exercise because of conflict with her work schedule. Twenty-nine subjects underwent the screening work-up but did not follow-up and did not respond to telephone follow-up. One hundred eighteen subjects were then qualified and included in the study. All the subjects deemed qualified to be included in this study were able to undergo the test. The demographic, and anthropometric characteristics of the subjects per stratum are shown in Tables 1 and 2.

Table 1. Baseline Anthropometric data and Borg's scale score on Dyspnea of Subjects per Age Stratum.

\begin{tabular}{llllll}
\hline Age Group & Gender & Number of subjects & Weight $\mathbf{( k g )}$ & Height $(\mathbf{c m})$ & Borg's scale \\
\hline $20-29$ & Male & 15 & 62.64 & 167 & 5.08 \\
& Female & 15 & 49.65 & 154.67 & 5.08 \\
$30-39$ & Male & 14 & 61.7 & 165.3 & 3.5 \\
& Female & 14 & 50.62 & 152.9 & 3.75 \\
$40-49$ & Male & 16 & 68.2 & 167 & 4.1 \\
& Female & 14 & 53.8 & 155.1 & 3.31 \\
$50-60$ & Male & 13 & 63.6 & 164.9 & 4.05 \\
& Female & 17 & 52.35 & 151.6 & 1.9 \\
\hline
\end{tabular}

The exercise test was terminated most commonly because of leg pain. The mean Borg Scale for intensity of symptoms for each stratum is also shown in table 1 . The exercise test was terminated by the patient in all of the cases. No untoward events were noted during the conduct of all the tests.

Table 2. Comparison of Baseline Anthropometric and lung function data of Subjects Between Gender.

\begin{tabular}{|c|c|c|c|c|}
\hline & Men $\mathbf{n}=58$ & Women $n=60$ & Total $n=118$ & $P$ value \\
\hline Mean Age in years & $38.43(12.04)^{*}$ & $39.02(11.78)^{*}$ & $38.73(11.86)^{*}$ & 0.7899 \\
\hline Mean Weight in $\mathrm{kg}$ & $64.06(8.46)$ & $51.61(4.87)$ & $57.73(9.27)$ & $<0.001 * *$ \\
\hline Height in $\mathrm{cm}$ & $166.10(6.09)$ & 153.57 (5.29) & $159.73(8.47)$ & $<0.001 * *$ \\
\hline Mean FEV1 in L & $3.13(0.55)$ & $2.34(0.45)$ & $2.73(0.64)$ & $<0.001 * *$ \\
\hline Mean FVC in L & $3.65(0.659)$ & $2.70(0.52)$ & $3.17(0.76)$ & $<0.001 * *$ \\
\hline
\end{tabular}

*Values in parentheses are standard deviations

** $\mathrm{p}$ value is $<0.05$ and is statistically significant

There were significant differences in the weight, height, FEV1 and FVC between men and women in the study population with consistently higher values among male subjects (Table 2). There was no significant difference in the ages of the two groups.
Male subjects attained significantly higher workloads and maximal tidal volume than female subjects as seen in Table 3. There was no significant difference in $\mathrm{VO}_{2}$ peak, maximal respiratory rate, and maximal heart rate between men and women as seen in Table 3. 
Table 3. Cardiopulmonary responses at maximal exercise of subjects and according to gender.

\begin{tabular}{|c|c|c|c|c|}
\hline & Males $\mathbf{N}=\mathbf{5 8}$ & Females $\mathbf{N}=\mathbf{5 0}$ & Total & $P$ value \\
\hline WORKmax in Watts & $154.21(26.59)^{*}$ & $93.02(15.57)^{*}$ & $123.35(37.59)^{*}$ & $<0.001$ \\
\hline $\mathrm{VO}_{2}$ peak in $\mathrm{L} / \mathrm{min}$ & $4.90(3.11)$ & $4.56(2.41)$ & $4.73(2.77)$ & 0.50 \\
\hline RRmax in breaths per minute & $25.87(5.79)$ & $24.89(3.84)$ & $25.38(4.91)$ & 0.29 \\
\hline HRmax in beats per minute & $160.19(16.61)$ & $153.72(20.72)$ & $156.98(18.96)$ & 0.067 \\
\hline Respiratory exchange ratio (RER) & $0.62(0.37)$ & $0.37(0.17)$ & $0.49(0.31)$ & $<0.001$ \\
\hline
\end{tabular}

*Values in parentheses are standard deviations

WORKmax=Maximum Working Capacity

$\mathrm{VO}_{2}$ peak=Peak oxygen uptake

VTmax $=$ Maximal Tidal Volume

RRmax $=$ Maximal respiratory rate

HRmax=Maximal heart rate

The predictive equations for normal values for Maximum Working Capacity, peak $\mathrm{VO}_{2}$, peak $\mathrm{VCO}_{2}$ and TV for the Filipino population are shown below:

$$
\text { TVpeak }=(-1.564)+(1.559 \mathrm{E}-02 \times \mathrm{Wt} .)+(0.196 \mathrm{x} \mathrm{sex})+(1.271 \mathrm{E}-02 \times \mathrm{Ht} .)
$$

$\mathrm{R}=0.761$ Rsquared $=0.579$ Adjusted Rsquared $=0.567$

$\mathrm{K}-\mathrm{S}$ (Test of Normality) is not significant

Levene's Test for equality of error variances is not significant

Sex: male $=1$ female $=0$

$$
\log \left(\mathrm{VCO}_{2} \text { peak }\right)=(-6.482 \mathrm{E}-02)+(0.158 \times \mathrm{sex})+(5.476 \mathrm{E}-03 \times \mathrm{Wt} .)-(2.437 \mathrm{E}-03 \times \mathrm{Age})
$$

$\mathrm{R}=0.805$ Rsquared $=0.648$ Adjusted Rsquared $=0.639$

$\mathrm{K}-\mathrm{S}$ (Test of Normality) is not significant

Levene's Test for equality of error variances is not significant

Sex: male $=1$ female $=0$

$$
\log (\text { WORKmax })=(1.772)+(0.162 \times \text { sex })+(4.636 \mathrm{E}-03 \times \mathrm{Wt} .)-(1.277 \mathrm{E}-03 \mathrm{x} \text { Age })
$$

$\mathrm{R}=0.853$ Rsquared $=0.728$ Adjusted Rsquared $=0.720$

$\mathrm{K}-\mathrm{S}$ (Test of Normality) is not significant

Levene's Test for equality of error variances is not significant

Sex: male $=1$ female $=0$

$$
\mathrm{VO}_{2} \text { peak }=(-3.104)-(6.967 \mathrm{E}-02 \mathrm{xAge})-(0.558 \mathrm{xsex})+(6.713 \mathrm{E}-02 \mathrm{xHt})+(1.457 \mathrm{E}-03 \mathrm{x} \mathrm{Wt})
$$

$\mathrm{R}=0.350$ Rsquared $=0.123$ Adjusted Rsquared $=0.092$

Sex: male $=1$ female $=0$

\section{Discussion}

Age was not a statistically significant variable in the determination of the VT peak equation. The correlation coefficient for the VT peak equation was 0.579 . Both K-S test for normality and Levene's test for equality of error variances for the VT peak formula were not significant. This implies that the power of the derived VT peak equation is statistically acceptable.

The predictive equation for $\mathrm{VCO}_{2}$ peak required logarithmic transformation to improve its power. Height was not a statistically significant predictor of $\mathrm{VCO}_{2}$ peak. The $\mathrm{R}$ squared for the derived model for $\mathrm{VCO}_{2}$ peak was 0.648 . Both K-S test for normality and Levene's test for equality of error variances were also not significant. Again, the above results makes the power of the $\mathrm{VCO}_{2}$ peak predictive equation statistically acceptable.

The predictive equation for Work max also required logarithmic transformation. Height was also not a statistically significant predictor of Work max. R squared was 0.728 . Both K-S test for normality and Levene's test for equality of error variances were again also not significant. The Work max predictive equation had the best correlation coefficient among all the formulae derived in this study.

No transformation was needed for the candidate predictors in all of the above models.

From the partial regression plots, sex, height, and weight are not helpful in regressing $\mathrm{VO}_{2}$ peak when all other variables are already in the model. Age seems to be somewhat helpful as there was note of a small but statistically significant negative correlation with $\mathrm{VO}_{2}$ peak. From the coefficient of multiple determination, $\mathrm{R}$ squared, the variation of $\mathrm{VO}_{2}$ peak that can be explained by age, sex, height and weight simultaneously is 
only 0.123 . The performance of the predictive model $\mathrm{VO}_{2}$ peak may be due to several possibilities. First, the variables considered may not be really predictive of $\mathrm{VO}_{2}$ peak. Important variables may have been missed including those related to time. A second possibility is that the sample size was not enough to derive a more powerful formula. Care was taken in ensuring that the subjects would attain their maximum capacity to exercise in that the criteria for maximum exercise were met and that the formula derived for Work max was statistically powerful, hence, this possibility was not considered as a reason for the less than optimal performance of the $\mathrm{VO}_{2}$ peak formula. As Jones [6] has written, "the precision of most normal values in physiology is seldom better than \pm 20 per cent, perhaps we should not attempt to improve on these approaches but live with the variability."

Table 4. Comparison of the mean absolute percentage error using the predictive equation for maximal oxygen uptake and maximal work rate from the present study and previously published studies.

\begin{tabular}{|c|c|c|}
\hline & WORK max* & $\mathrm{VO}_{2}$ peak $^{* *}$ \\
\hline Present study & $2.65 \%$ & $48.87 \%$ \\
\hline Ong, et $\mathrm{al}^{11}$ & $9.26 \%$ & $7.59 \%$ \\
\hline Jones, et $\mathrm{al}^{6}$ & $39.98 \%$ & $50.61 \%$ \\
\hline
\end{tabular}

*WORK $\max =$ maximal work rate at maximal exercise

$* * \mathrm{VO}_{2}$ peak=peak oxygen uptake at maximal exercise

We then attempted to compare the published prediction equations using the CPET results obtained from our subjects. Jones' formulae for deriving normal values are considered the most replicable in the Caucasian population and Ong et al's equations derived from Chinese adults had comparable correlation coefficients with other established formulae $[6$, $11,12]$. Ong's study, they made the conclusion that estimates made from prediction equations derived from Caucasian populations indeed tended to overestimate actual values from Asian populations [11]. Using the CPET results obtained from our patients, the Mean Absolute Percentage Error (MAPE) for predictive equations for Work max and $\mathrm{VO}_{2}$ peak from Jones' and Ong's studies were derived (Table 4). The predictive equations of Ong, et al performed better than the formula derived by Jones, et al $[6,11]$. This may reflect the race-related differences in exercise capacity. This study had a better fit than Ong's study in the prediction equation for Work max because this study's population was used to compare the two equations [11]. However, for the $\mathrm{VO}_{2}$ peak prediction equation, Ong's formula had a better performance compared to the equation in this study while this study's equation performed as well as Jone's formula [6, 11]. Thus, the Filipino subject probably performs in a similar manner with a Chinese subject and that the prediction equation of Ong et al for $\mathrm{VO}_{2}$ peak may also be utilized for the Filipino population [11]. The null hypothesis was was rejected in this study since differences between the exercise testing formulae for Caucasians and the Filipino subjects in this study were noted. For accuracy in interpreting exercise testing results, it is worthwhile to utilize predictive equations to derive normal values specific for a certain racial population.

It must be emphasized that the performance in an exercise test heavily depends on the protocol used for exercise so that the predictive equations in this study can be used only for exercise tests using a similar protocol.

The authors realize the need to validate the results in this study with another sample of the normative Filipino population.

\section{Conclusion}

Cardiopulmonary exercise testing was performed on a community based, healthy, sedentary Filipino population using a standard maximal incremental cycle ergometry protocol. Predictive equation models were derived for Maximum Working Capacity, peak $\mathrm{VO}_{2}$, peak $\mathrm{VCO}_{2}$, and tidal volume during exercise. The derived predictive formulae performed as well as other published predictive equations.

\section{Conflict of Interest Notification}

All the authors declare no potential conflict of interest as regards this study. None of the authors hold any position, personal relationship, nor have any financial investment in equipment nor companies related to cardiopulmonary exercise testing.

\section{References}

[1] Mahler DA, Horowits MB. Clinical Evaluation of Exertional Dyspnea. Clinics in Chest Medicine, 1994, 15 (2): 259-69.

[2] Wasserman K, Hansen JE, Sue DY, et al. Principles of exercise testing and interpretation. J Cardiopulm Rehabil Prev, 1987, 7 (4): 189.

[3] The American Thoracic Society/American College of Chest Physicians guideline. Statement on cardiopulmonary exercise testing. Am J Respir Crit Care Med, 2003, 167 (2): 211-77.

[4] Hansen J and Wasserman K. Exercise Testing. In: Leslie Chusid, The Selective and Comprehensive Testing of Adult Pulmonary Function. Philadelphia, WB Saunders, $1^{\text {st }}$ ed, 1983, 199-224.

[5] Balgos AA, Lua L, Pascual R. Cardiovascular and Respiratory Adjustments in Normal Volunteers during Modified Exercise Tests in Comparison to Standard Exercise Tests. Respirology, 1996, 1; 55-60.

[6] Jones NL. Clinical Exercise Testing. $3^{\text {rd }}$ ed. Philadelphia, WB Saunders Co., Harcourt-Brace Jovanvich Inc., 1988, 1-3; 123-9.

[7] Paap D, Takken T; Reference values for cardiopulmonary exercise testing in healthy adults: a systematic review. Expert Rev Cardiovasc Ther, 2014, 12 (12): 1439-1453.

[8] Pothoff G, Winter U, Wasserman K. Ergospirometric Studies of Normal Probands for an Unsteady State Increment Test Program. Kardiology, 1994, 82 (2): 116-123. 
[9] Rochmis, P. \& Blackburn, H. Exercise Test. A Survey of Procedures, Safety and Litigation Experience in Approximately 170,000 Tests. JAMA, 1971, 17 1061-1066.

[10] McKelvie R and Jones NL. Cardiopulmonary Exercise Testing. Clinics in Chest Medicine, 1989, 10; 277-291.
[11] Ong K, Loo C, Ong Y, et al. Predictive Values for Cardiopulmonary Exercise Testing in Sedentary Chinese Adults. Respirology, 2002, 7: 225-231.

[12] Jones N, Makrides L, Hitchcock C, et al. Normal Standards for an Incremental Progressive Cycle Ergometer Test. American Review of Respiratory Diseases, 1995, 131; 700-708. 